\title{
Electrical Characterization of Electronic Materials Using FIB-assisted Nanomanipulators
}

*Correspondence to:

Hwang $\mathrm{J}$

Tel: +82-2-320-3069

Fax: +82-2-333-0127

E-mail: jhwang@wow.hongik.ac.kr

Received December 4, 2012

Revised December 6, 2012

Accepted December 6, 2012

\author{
Jae-Hong Roh, Yil-Hwan You ${ }^{1}$, Jae-Pyeong Ahn, Jinha Hwang ${ }^{1, *}$ \\ Nano-analysis Center, Korea Institute of Science \& Engineering, Seoul 136-791, Korea \\ ${ }^{1}$ Department of Materials Science \& Engineering, Hongik University, Seoul 121-791, Korea
}

cher

Focused Ion Beam (FIB) systems have incorporated versatile nanomanipulators with inherent sophisticated machining capability to characterize the electrical properties of highly miniature components of electronic devices. Carbon fibers were chosen as a model system to test the applicability of nanomanipulators to microscale electronic materials, with special emphasis on the direct current current-voltage characterizations in terms of electrode configuration. The presence of contact resistance affects the electrical characterization. This resistance originates from either i) the so-called "spreading resistance" due to the geometrical constriction near the electrode - material interface or ii) resistive surface layers. An appropriate electrode strategy is proposed herein for the use of FIB-based manipulators.

Key Words: Focused ion beam, Nanomanipulators, Spreading resistance, Electrode configuration

\section{INTRODUCTION}

The ubiquitously-digitized information and communication systems in these days demand high-performance electronic devices in which nano- and microscale components are integrated using passive and active elements on mostly Si or glass substrates. Although device elements have been constructed in nano- and microscale, discrete electrical and structural characterizations and evaluation of functioning of each element are required to optimize the electrical and dielectric performance and to evaluate functional failure and defect origins for these devices.

A focused ion beam (FIB) system offers versatile applications in materials research and development owing to the inherent capability of high-precision milling (Giannuzzi et al., 1998). It is used for in situ nanoscale characterization, fabrication, patterning, and intentional manipulation of electronic devices (Nikawa, 1991; Nam et al., 2005; Tseng, 2005; Lasagni et al., 2008; Jeon et al., 2010). Among the auxiliary attachments, nanomanipulators uniquely allow in situ electrical characterization in combination with high-magnification imaging, e.g., scanning electron microscopy (SEM). However, there are issues to be answered for extensive applications of FIB-assisted manipulators. In particular, contact issues occur at the electrode - material interface, involving unreasonably high resistance and poor reproducibility.

In this paper, we report the application of FIB-assisted nanomanipulators for electrical characterization of microscale structures and materials. Carbon fibers were chosen as a model system to test the applicability of nanomanipulators to microscale systems, with special emphasis on the direct current (DC) current-voltage characterizations. The model system was monitored in terms of the electrode configurations; two-, three-, four-point configurations were used with the manipulators. Depending on the multipoint electrode structure, the contact-related portions can be deconvoluted from the integrated bulk-based electrical information. Using the results from the present case study

This research was supported by the Converging Research Center Program (2011K000607), Pioneer Research Center for Nano-morphic Biological Energy Conversion and Storage, Basic Science Research Program (2010-0009179) through the National Research Foundation of Korea (NRF) funded by the Ministry of Education, Science and Technology.

@ This is an open-access article distributed under the terms of the Creative Commons Attribution Non-Commercial License (http://creativecommons.org/licenses/by-nc/3.0) which permits unrestricted noncommercial use, distribution, and reproduction in any medium, provided the original work is properly cited.

Copyright (C) 2012 by Korean Society of Microscopy 
on carbon microfibers, the geometrical contact resistances were estimated numerically, which was crucial for applying the nanomanipulators to microscale material and structure characterization. We will discuss the unique capabilities of FIB-assisted nanoprobes along with the concurrent limitations. The basic information on FIB-based probes should be of special interest to potential users specializing in electronic devices.

\section{MATERIALS AND METHODS}

After organic dilution to optimize the aerial density of the carbon microfibers, the fibers were dispersed onto $\mathrm{SiO}_{2}$ thin films that were thermally grown on $\mathrm{Si}$ wafers. The $\mathrm{SiO}_{2}$ thin film served as an insulating layer for electrical characterization in a conducting or semiconducting material system. Electrical characterizations were performed using nanomanipulators (MM3A EM; Kleindiek Nanotechnik GmbH, Reutlingen, Germany) installed in an SEM-FIB system (Qunata 3D; FEI Company, Eindhoven, The Netherlands) with a semiconductor device analyzer (B1500A; Agilent Technologies, Palo Alto, CA, USA). The positions of the nanomanipulators were controlled with a $5 \mathrm{~nm}$ resolution in combination with contact-sensing capabilities. The electrical connections were employed in two-, three-, or four-electrode configurations with positional flexibilities among the four contact points using FIB-aided manipulators. The present work collects the corresponding electrical data based on DC-based currentvoltage characteristics, taking into account the compliance limitations encountered in data acquisition.

\section{RESULTS AND DISCUSSION}

Fig. 1A shows the microstructure of the carbon microfibers dispersed on the insulating $\mathrm{SiO}_{2}$ thin films deposited on $\mathrm{Si}$ wafers. Depending on the solid loading of the dilute solution in which the carbon fibers were mixed with a matrix of an organic solvent, the carbon fibers were interconnected randomly within the three-dimensional network. Since the electrical function of the overall carbon-felt material system, i.e., a non-woven cloth made up of carbon fibers, was critically dependent on the individual carbon fibers, a single carbon fiber had to be characterized without ambiguity in terms of its electrical characteristics, especially conductivity (or its reciprocal, resistivity). After appropriate positioning of the carbon microfibers, a single carbon microfiber was selected for FIB-based electrical analysis (Fig. 1B). Electrical contacts were then formed between the nanomanipulators and the
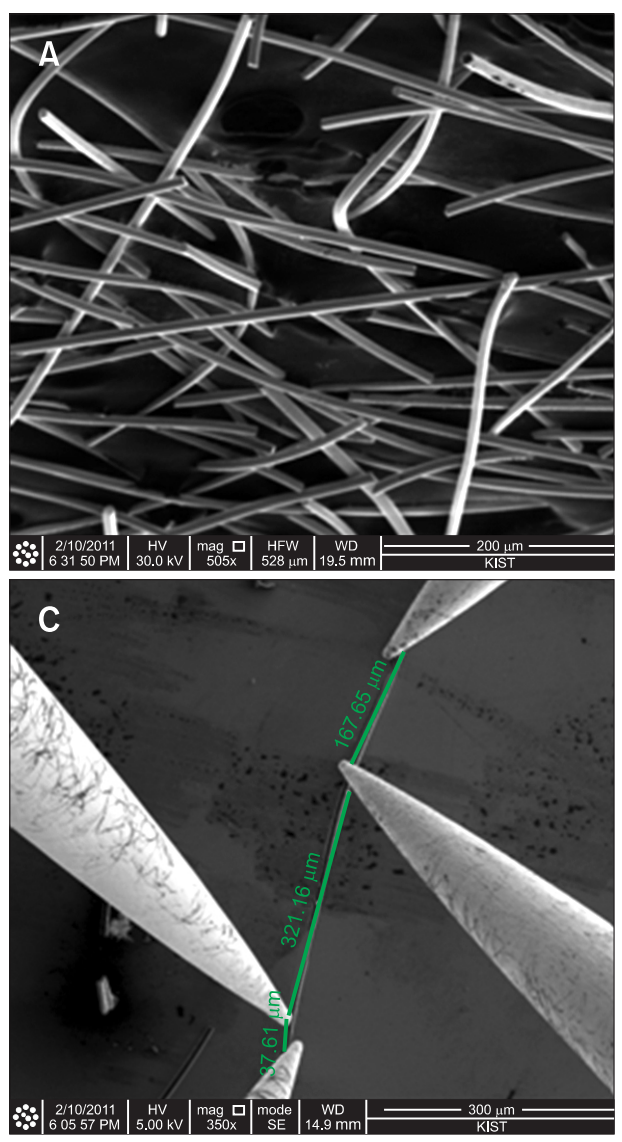

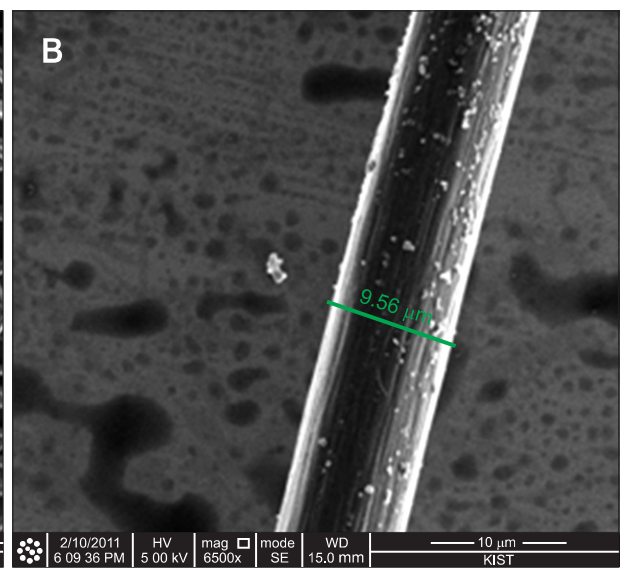

Fig. 1. Microstructure of carbon microfibers at (A) low magnification, (B) high magnification, and (C) a four-point electrode configuration (diameter $=9.56 \mu \mathrm{m}$; length $=321.16 \mu \mathrm{m}$ ). 
carbon fiber at four different locations, as shown in Fig. 1C. In particular, intentional combinations were formed among four contact points, allowing for two-, three-, and four-point electrode configurations.

Fig. 2A-C show the two-, three-, and four-point electrode resistance measurements, respectively. The measured threepoint electrode configuration resistance was $3.6 \times 10^{2} \mathrm{ohm}$ and the two-point electrode resistance was approximately $5.4 \times 10^{4} \mathrm{ohm}$ (See Fig. 2A and B). However, the four-point electrode configuration resistance was calculated as $49 \mathrm{ohm}$, indicating there were no contact issues with the electrodes (Fig. 2C). In the two- and three-point electrode configurations, the relationship between the current and voltage was highly linear, implying that no non-ohmic effects existed in the carbon fiber positioned using nanomanipulators, as shown in Fig. 1C. Therefore, the remaining issue was limited only to the geometrical constriction between the electrodes and the material. The values of the two- and three-point electrode configuration resistance reflected the resulting contribution of electroding interfaces where the two-point resistance involved two interfacial components, unlike the single interface
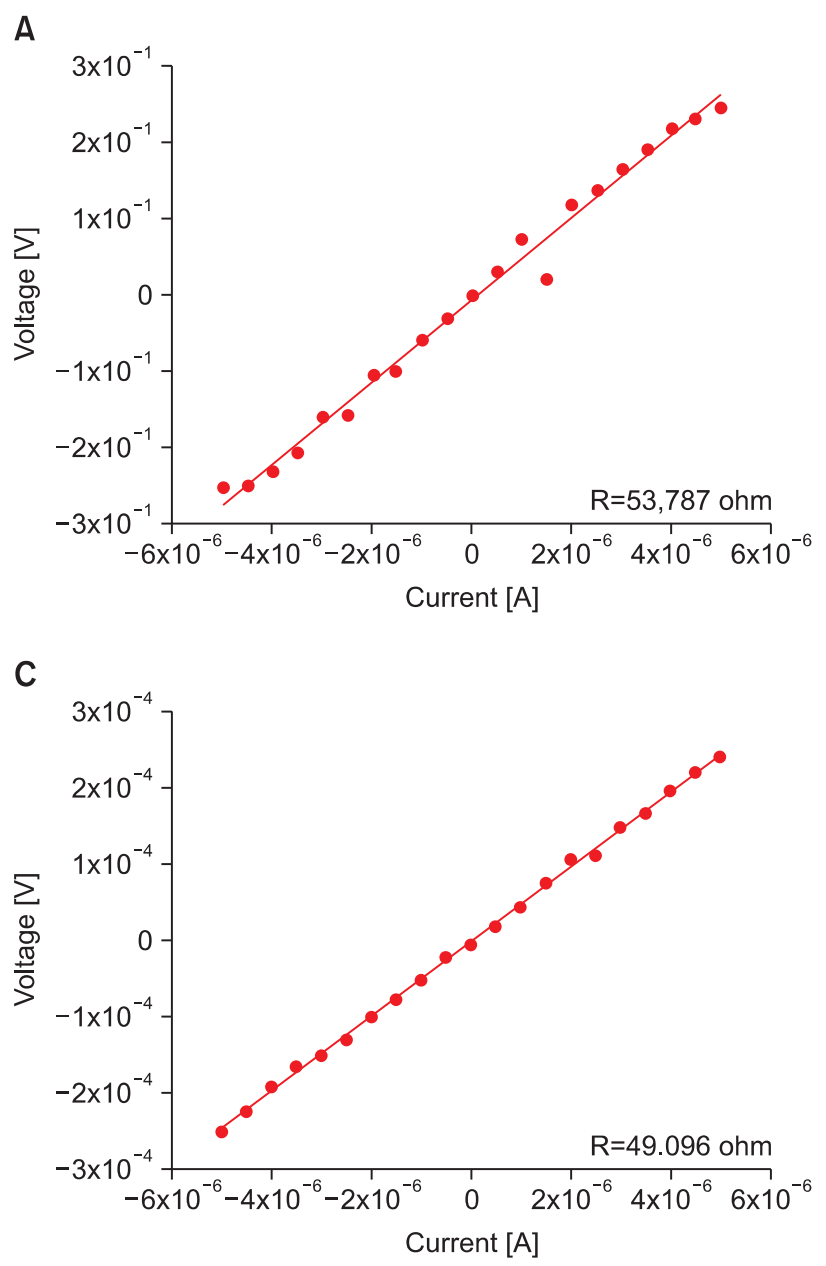

involved in the three-point electrode measurements. Such large discrepancy can be associated with the geometrically limited contact resistances at the probing interfaces (Newman, 1966; Hwang et al., 1997).

Depending on the electrode configurations, the corresponding current-voltage characteristics can involve the undesired effect of geometrically limited contact resistance present at the metal-carbon interfaces. The corresponding equivalent circuit is shown in Fig. 3. At every contact point between the electrode and the material, site-specific contact issues existed, originating from the geometrically constricted interfaces (Fig. 4). The spreading resistance $R_{\text {spreading }}$ is determined by the equation (Newman, 1966)

$$
\mathrm{R}_{\text {spreading }}=1 /(4 \sigma \mathrm{a})
$$

where $\mathrm{a}$ is the contact radius and $\sigma$ is the conductivity of the material. Furthermore, the contact resistance is inversely proportional to the contact radius. The values of the contact resistance was not always be identical among the contact points, leading to significant variations in the position of

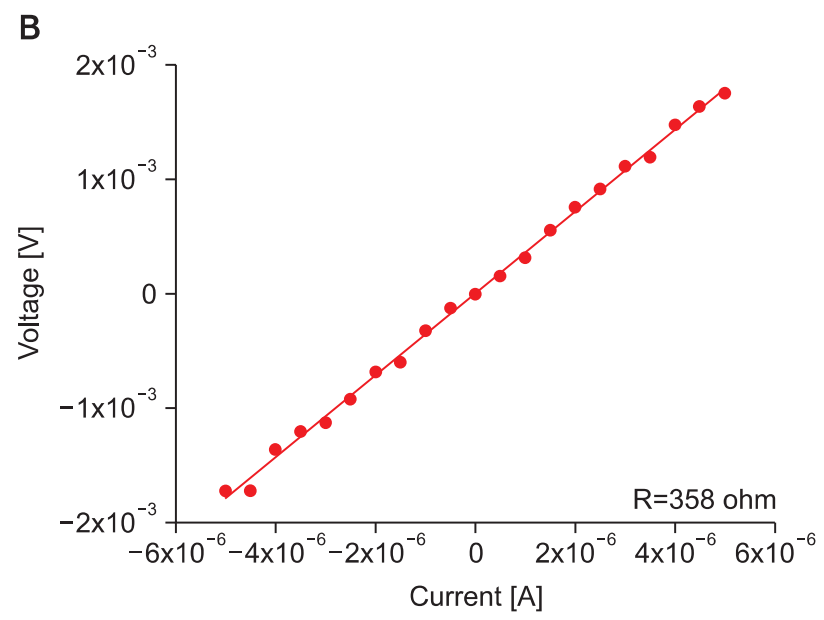

Fig. 2. Current-voltage characteristics at a (A) two-point electrode configuration, (B) three-point electrode configuration, and (C) four-point electrode configuration. 


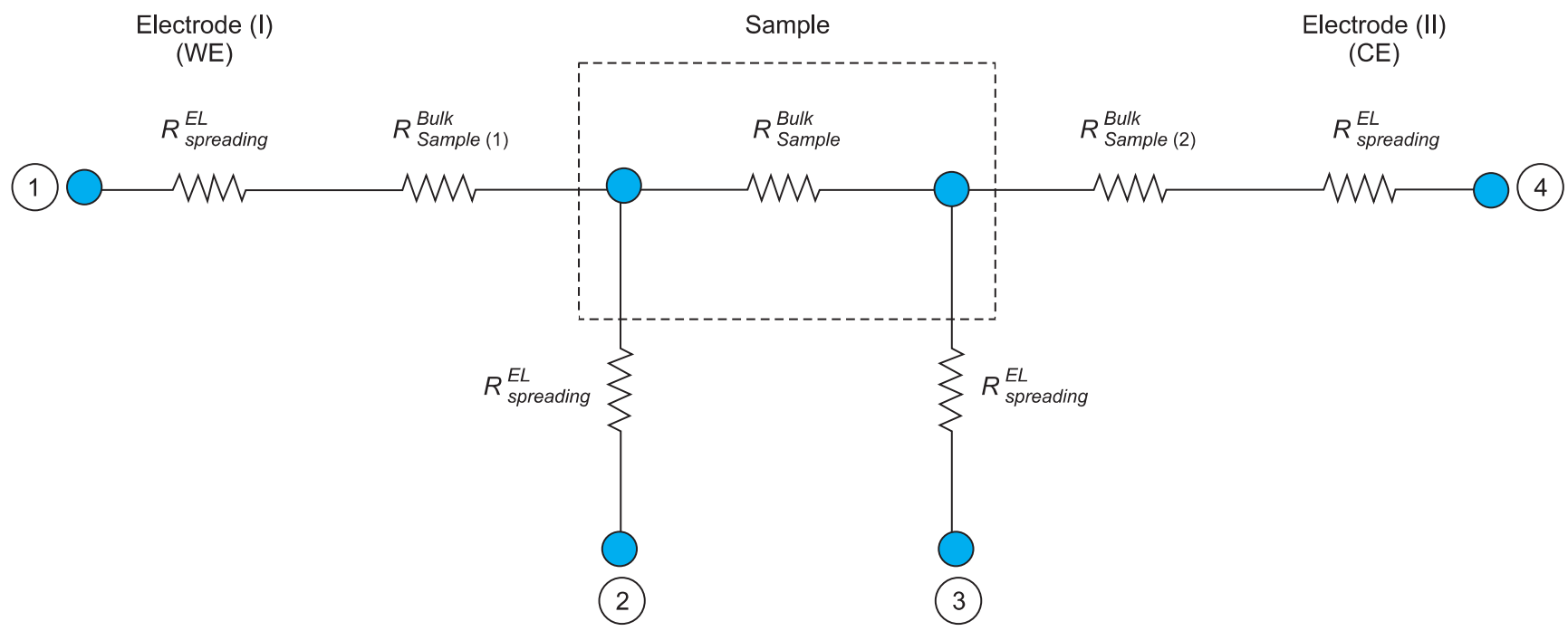

Fig. 3. Equivalent circuit description of a multi-electrode configuration employed for direct current-based current-voltage characteristics. WE, working electrode; CE, counter electrode.

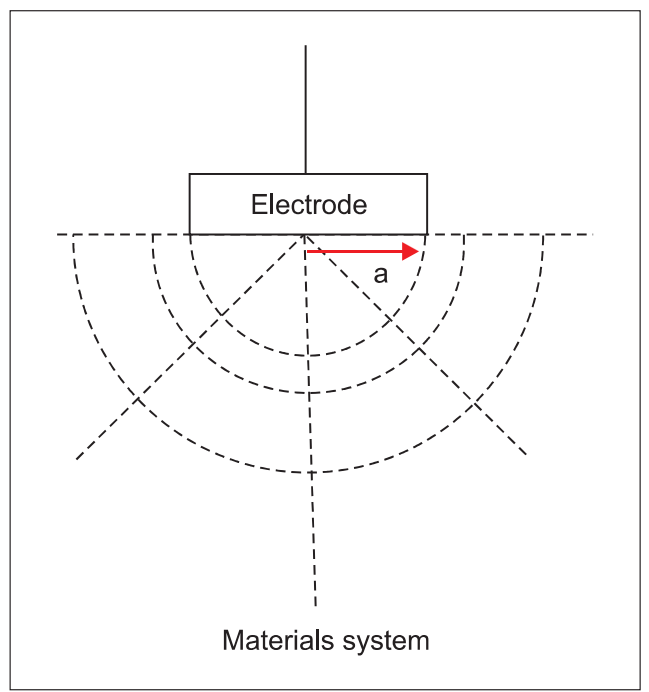

Fig. 4. Spreading resistance in an infinite material system for a geometrically constricted electrode.

contact formed between the probing tip and the carbon fiber. Surprisingly, the calculated contact radius was located in the sub-angstrom regime. The small value did not match the physical observation on the contact area. Accordingly, it appears that additional resistive materials were located between the metallic tip and carbon fiber, in the form of surface contamination or oxidation layers. Depending on the magnitude of the applied electric field, those materials will function either as a barrier or a tunneling layer.

Therefore, the direct contact of FIB-manipulators should be scrutinized before quantitative estimation of the material's resistivity (or conductivity). The four-point electrode configuration led to a resistivity of $1.1 \times 10^{-5} \mathrm{ohm} \cdot \mathrm{m}$, excluding the contact resistance and the effect of undesired surface layers, since the four-point electrode configuration can extract only the potential difference between the two sensing points. The calculated values were in excellent agreement with values in the range 0.91 to $1.38 \times 10^{-5} \mathrm{ohm} \cdot \mathrm{m}$. In other words, the effectiveness of FIB-based nanomanipulators should be guaranteed after empirical verification of contactbased problems. Otherwise, simplified two-point electrode configuration can mislead the numerical estimation of material properties such as resistivity. Our next paper will report the characterization and structure of nanoscale materials.

\section{CONCLUSIONS}

The spreading resistance originated from the geometrical constriction between a nano-manipulator and a single microscale carbon fiber. In addition to the spreading resistance, there were indications of the existence of resistive barriers (in the form of surface contamination or oxidation layers) between the probing tip and the carbon fiber. The two- and three-electrode configurations suffered from contact problems present because of spreading resistance effects and the spurious surface layers. Only the four-point electrode configuration could eliminate the misleading effect associated with contact issues between the electrodes and the conducting probe. According to the four-point electrode configuration measurement, the calculated resistivity of a microscale carbon fiber was in excellent agreement with the reported bulk value for carbon materials. Therefore, FIB- 
based nanomanipulators should be employed in electrical and dielectric characterizations of nanoscale and micro- scale systems only after the electrode-related phenomenon is understood.

\section{REFERENCES}

Giannuzzi L A, Drown J L, Brown S R, Irwin R B, and Stevie F A (1998) Applications of the FIB lift-out technique for TEM specimen preparation. Microsc. Res. Tech. 41, 285-290.

Hwang J H, Kirkpatrick K S, Mason T O, and Garboczi E J (1997) Experimental limitations in impedance spectroscopy: Part IV. Electrode contact effects. Solid State Ionics 98, 93-104.

Jeon J, Floresca H C, and Kim M J (2010) Fabrication of complex three-dimensional nanostructures using focused ion beam and nanomanipulation. J. Vac. Sci. Technol. B 28, 549-553.

Lasagni F, Lasagni A, Engstler M, Degischer H P, and Mucklich F (2008) Nano-characterization of cast structures by FIB-tomography. Adv.
Eng. Mater. 10, 62-66.

Nam C Y, Kim J Y, and Fischer J E (2005) Focused-ion-beam platinum nanopatterning for GaN nanowires: ohmic contacts and patterned growth. Appl. Phys. Lett. 86, 193112.

Newman J (1966) Current distribution on a rotating disk below the limiting current. J. Electrochem. Soc. 113, 1235-1241.

Nikawa K (1991) Applications of focused ion beam technique to failure analysis of very large scale integrations: a review. J. Vac. Sci. Technol. B 9, 2566-2577.

Tseng A A (2005) Recent developments in nanofabrication using focused ion beams. Small 1, 924-939. 